\title{
Improving the Accuracy of Environment-specific Vehicular Channel Modeling
}

\author{
Xiaohui Wang $^{\dagger}$, Eric Anderson ${ }^{\dagger}$, Peter Steenkiste ${ }^{\dagger}$, and Fan Bai ${ }^{\star}$ \\ + Carnegie Mellon University \\ Pittsburgh, PA, USA \\ ${ }^{*}$ Electrical \& Controls Integration Lab \\ General Motors Global R\&D, Warren, MI, USA
}

\begin{abstract}
Networking research benefits from the controlled, repeatable experimentation provided by simulation and emulation systems. Making such simulations realistic is a challenge for wireless systems and is especially difficult for vehicular networks. This paper introduces a realistic vehicular channel simulation model that includes a Line-Of-Sight $(L O S)$ module and Vehicle-to-Vehicle (V2V) fading module specially designed for vehicular channels.

Specialized models can provide a close approximation of real channel conditions, but their accuracy is limited by the quality of input information about the environment being modeled. In this paper, we present a systematic approach to estimate location-specific scattering properties using aerial photography. We show that this approach significantly improves the accuracy of simulated channel fading.
\end{abstract}

\section{Categories and Subject Descriptors}

I.6.5 [Simulation and Modeling]: Model DevelopmentModeling methodologies

\section{Keywords}

Vehicular Channel Modeling, Fading Model, Doppler Spectra, Model Parameter Estimation

\section{INTRODUCTION}

Radio channel properties are significant, variable, and difficult to estimate for vehicle-to-vehicle (V2V) and vehicle-toinfrastructure (V2I) wireless communication. Vehicles can travel through very different environments, producing distinctly different channels in e.g. urban centers, rural roads, and multi-lane highways. Even within a given area, the location and density of surrounding objects varies dramatically, leading to varying impact on reflected signals.

Despite - or even because of - these challenges, it is useful to model and simulate channels in vehicular networks in a controllable platform. Realistic vehicular channel models

Permission to make digital or hard copies of all or part of this work for personal or classroom use is granted without fee provided that copies are not made or distributed for profit or commercial advantage and that copies bear this notice and the full citation on the first page. To copy otherwise, to republish, to post on servers or to redistribute to lists, requires prior specific permission and/or a fee.

WiNTECH'12, August 22, 2012, Istanbul, Turkey.

Copyright 2012 ACM 978-1-4503-1527-2/12/08 ...\$15.00. provide a basis for analysis and evaluation of wireless vehicular networks by allowing flexible, controllable, repeatable experimentation. While general mobile-to-mobile channel models are currently supported in some wireless network simulation and emulation systems [1-3], those models do not capture the unique and highly dynamic properties of vehicular channels.

The gap between vehicular channels and general mobileto-mobile channel models lies in: (a) highly variable blockage of Line-Of-Sight $(L O S)$; and (b) location-specific scatterer distribution and corresponding fading effects. The direct $L O S$ path between two communicating vehicles exits when there is no other cars travel in between, and may be easily interrupted by lane merging of intervening vehicles. Current simulation platforms often overlook the impact and no direct models handle the $L O S$ effects explicitly.

Fading effects, which are caused by scatterers (objects that reflect signals) in the environment, are location-specific in the vehicular network. Unlike general mobile-to-mobile wireless channel models, where scatterers are stationary objects and do not change over time, vehicular networks have a unique set of varying scatterers. These include road-side 'stationary' scatterers, such as trees and buildings, and moving vehicles as smaller 'mobile' scatterers.

Although road-side scatterers are stationary, their density and location do change over space as vehicles drive by. Therefore, 'stationary' scatterers create time-varying impacts on channel fading properties. Recent research [4] proposed a geometrical channel modeling of fading effects originated from 'stationary' road-side scatterers. While the model reflects $\mathrm{V} 2 \mathrm{~V}$ specific fading feature, it requires extra information on scatterer distribution along routes. Without accurate parameter values, modeling of fading effects is estimated, losing the accuracy targeted at any specific location.

This paper presents our design of a realistic vehicular channel simulation model that captures the unique channel features in V2V communication. We will first introduce the general simulation architecture, and then focus specifically on accurate modeling of environment-specific vehicular channels. Using previous work on flexible channel emulation [3], we were able to develop new channel components to represent unique vehicular channel properties addressed above:

- A V2V LOS simulation model that represents vehicleto-vehicle $L O S$ blocking features.

- A flexible and realistic V2V fading simulation model that represents fading effects from stationary scatterers. 
Meanwhile, we introduce our novel approach of estimating model parameter values from aerial maps. Road-side objects can thus be identified with high accuracy at any given location. Both channel frequency response and packet level performance are evaluated, and a trace-based study shows significantly improvement in parameter accuracy and channel modeling accuracy.

\section{A REALISTIC V2V SIMULATION MODEL}

Figure 1 shows our general architecture for wireless channel simulation and emulation. From top to down, it includes three major components: A world model, channel model control and channel model implementation, considering time scales from almost static to $\mu \mathrm{s}$. Comparing to previous wireless simulation models, two major components have been added to capture unique vehicular channel features. A Geo-spatial model is introduced to model finegrained environmental variation, and a LOS channel model component is added to reflect V2V LOS blocking properties. One component, the channel fading model, has been significantly expanded and enhanced to capture the effects of small-scale spatial variation in vehicular networks. The following discussion will zoom in to the three major parts of the general architecture (from bottom to top), with special focus on modeling vehicle-to-vehicle channels.

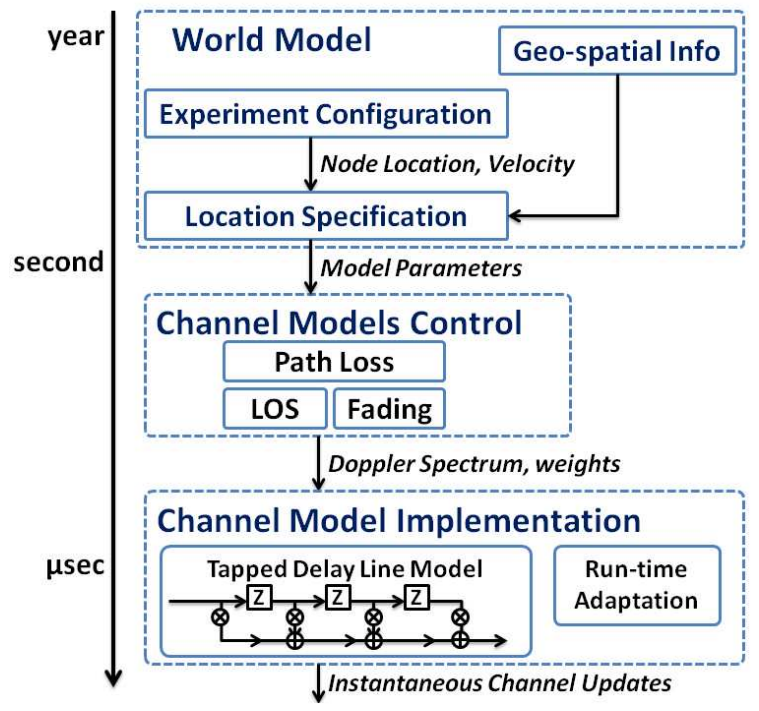

Figure 1: General Simulation Architecture

\subsection{Channel Model Implementation}

We briefly describe the channel model implementation framework that we use as the starting for our realistic channel simulation. The framework operates at two levels.

\subsubsection{Tapped Delay Line Model}

At the bottom level, the channel response is modeled in the time domain using a tapped delay line model. Each tap effectively represents a resolvable propagation path, and the evolution of each tap (path) weight provides a statistical approximation of Doppler spreading and non-resolvable multipath effects. The delay between paths and their relative magnitude determines the frequency selectivity of the channel and the degree of inter-symbol interference experienced.

\subsubsection{Run-time Adaptation}

The second level must generate the time-varying tap (path) weights to reflect desired large-scale attenuation and smallscale fading characteristics. The rate at which these tap weights change and the patterns of those changes determine coherence time, the (simulated) Doppler spreading, and higher-order statistics such as the average fade duration. Viewed in the frequency domain, the important properties of a tap weight sequence are captured by the Doppler power spectral density (Doppler spectrum) it produces. We therefore describe tap weight generation process in terms of generation of the fading spectrum for each path.

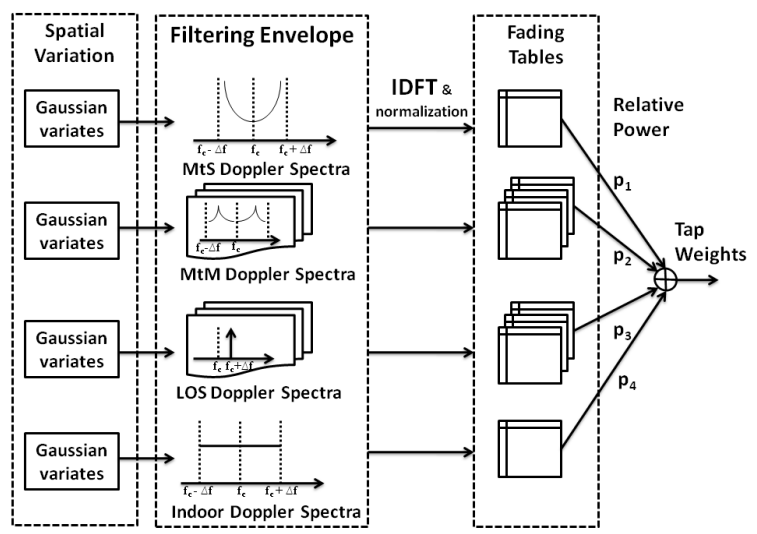

Figure 2: Tap weights generation process for a single path.

In order to support the dramatically varying tap fading spectra associated with a wide range of wireless channels, we use the architecture shown in Figure 2 to calculate each tap weight. The desired spectrum is defined as a weighted sum of simpler spectra (shown as Filtering Envelope), each representing a different type of scatterer or LOS (with similar delays that cannot be resolved).

A key feature of the model is that the parameters can capture a wide range of channel properties and can be modified at runtime, as signal propagation conditions change during an experiment. To reduce computational work at run time, fading tables can be generated off-line to represent predefined Doppler spectra, and adapted on-line using very lightweight operations. More details on the model can be found in [3].

\subsection{Channel Model Control in Vehicular Chan- nels}

We now introduce the vehicular channel model that we will use throughout the paper. The model is suitable for vehicle-to-vehicle communication in an urban/suburban environment, although the methodology we present in the next section is more general, i.e. it can be used for other vehicular models and outdoor environments as well. We chose this particular environment because there is growing interest in vehicular networking and it is also a challenging channel model because of the complexity of the environment, and rapid variation in channel conditions.

In typical urban/suburban vehicular networks, the two primary paths are line of sight (LOS) and reflections off buildings and possibly other objects (e.g. trees) lining the street. Since the differences in the two path lengths are rel- 
atively small, we only need to model a single resolvable path using two Doppler spectra, with one representing the contribution from LOS, and the other fading from reflections. Two types of fading in vehicular channels are modeled: (a) a geometry-based fading model for stationary scatterers; and (b) a fine grained fading model for mobile scatterers.

As shown in the Channel Model Control box in Figure 1, our $\mathrm{V} 2 \mathrm{~V}$ channel model includes three major components: (a) a large-scale path loss model; (b) a V2V LOS model; and (c) a small-scale fading model that represents time-varying reflection and scattering effects. Doppler spectra created in (b) and (c) are then combined in the tapped delay line model, as shown in Figure 2

The Doppler spectrum for the LOS component is determined by the relative velocity of the transmitting and receiving vehicles. Whenever there is no obstruction of propagation path between two vehicles, this Doppler spectrum component is added into the overall channel response. Reflections from both roadside objects (which are stationary) and other vehicles (moving or stopped) contribute to the fading effects. We refer to these objects as stationary scatterers and mobile scatterers.

Recent study on v2v channel [4] proposes a new approach to model the small-scale fading effects from reflections off roadside objects.

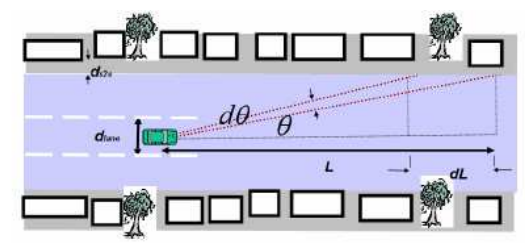

Figure 3: Geometrical Model for V2V Channel [4]

This fading model is a geometric model that uses location and density of roadside objects (buildings and trees) to estimate the reflections and their impact on fading, as shown in Figure 3. The assumption of scatterer location (arranged along both sides of the road) in this model is a close approximation of the reality in vehicular networks, thus we adopt this model as an example of geometry-based fading models for stationary scatterers.

In this model, the roadside objects are divided into small cones by the AOA (Angle Of Arrival of the reflected path. The fading Doppler spectrum is then computed by aggregating frequency response from scatterers within each small cone. More details can be found in the paper [4].

We do not attempt to specifically model the effects of mobile scatterers, primarily because we lack "ground truth" about other vehicles in our reference channel measurements, and it would be extremely difficult to validate our models. Rather, we argue that their effect is absorbed into our model of Doppler spectrum smoothness ( $\S 3.3$ ). Modeling mobile scatterers is not the focus of this paper, but we acknowledge the important role of mobile scatterers in fading channels.

\subsection{A Realistic V2V World Model}

In the simulation architecture, the World Model is a coarse representation of the physical world properties, and a set of rules for translating this information into channel model parameters. Then, time-varying vehicular channels can be created in simulation with parameters inputs that represent desired channel properties.

Vehicular channel models (e.g. $[4,5])$ require significant information about the environment, much of it specific to the exact locations of the communicating devices. In examining $\mathrm{V} 2 \mathrm{~V}$ channel measurements, we find that not only the observed channel conditions vary significantly within the same general environment, but a model using area-averaged parameter values performs significantly worse than the same model with best-estimated location specific values (see $\S 5$ ). It therefore does not suffice to have a good vehicular channel model - one must also have good knowledge of the environment, or sophisticated approach to generating synthetic environments in the $V 2 \mathrm{~V}$ World Model - to derive realistic channel conditions. This requires accurate estimation of geometry-based model parameters that are location-specific, and difficult to obtain, especially for considerably wide areas in a vehicular network.

We'll address the details in the next section.

\section{MODELS AND PARAMETERS}

There are three major sub-models to the vehicular channel model, as described in $\S 2.2$ : The line of sight Doppler spectrum $(\S 3.1)$, the geometry-based fading Doppler spectrum (§ 3.2$)$, and the "de-smoothing" of the Doppler spectrum (§ 3.3). Each of these sub-models has controllable parameters that can be tuned to reflect the time- and locationspecific effects within an environment. Additionally, the magnitude of each model's contribution is an environmentspecific property which must be modeled (§ 3.4).

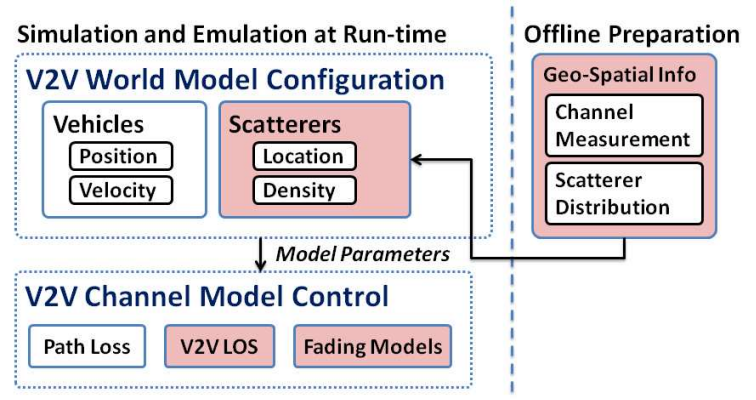

Figure 4: Determine parameter values for Channel Models

Generally, channel model parameters can be divided into two major categories: physical world parameters that directly represent physical world features, and signal propagation abstraction parameters that capture some effect of the physical world without representing the details that give rise to it. Either type can in principle be treated either as specific values or statistical distributions. Several example parameters are listed in Table 1 . Next, the parameter set of each specific $\mathrm{V} 2 \mathrm{~V}$ channel model component will be discussed.

\subsection{Line of Sight Doppler Spectrum}

The V2V LOS model decides when there exists a LOS between transmitting vehicle and receiving vehicle, and add

\footnotetext{
${ }^{1}$ Note that in any terrestrial environment, the path loss exponent is supposed to be a fitted mean path loss, but it is not generally treated as such.
} 


\begin{tabular}{|c|c|c|}
\hline & Physical World & $\begin{array}{l}\text { Signal } \\
\text { Propagation } \\
\text { Abstraction } \\
\end{array}$ \\
\hline Specific & $\begin{array}{l}\text { Topography } \\
\text { Structures } \\
\text { Location of mobiles } \\
\text { Velocity of mobiles }\end{array}$ & $\begin{array}{l}\text { Path loss exponent }{ }^{1} \\
\text { Clear line of sight } \\
\text { (LOS) }\end{array}$ \\
\hline Statistical & $\begin{array}{l}\text { Vegetation density } \\
\text { Traffic on roads } \\
\text { Building density } \\
\text { Terrain type }\end{array}$ & $\begin{array}{l}\text { Rician k-factor } \\
\text { Scatterer } \\
\text { distribution }\end{array}$ \\
\hline
\end{tabular}

Table 1: Examples of environment attributes / model parameters

corresponding Doppler spectrum component. LOS component exists as a dominant received signal when there is no other objects between communicating vehicles.

\subsubsection{LOS Doppler Spectra}

The Doppler effect on a line of sight signal between two (possibly) mobile stations is a simple frequency shift: If $\theta_{T x}$ $\left(\theta_{R x}\right)$ is the angle between the transmitter's (receiver's) velocity vector $\vec{v}_{T x}\left(\vec{v}_{R x}\right)$ and the direction of wave propagation, the Doppler shift $f_{d}$ is given by:

$$
\frac{f_{d}}{f_{0}}=\frac{\left|\vec{v}_{T x}\right|}{c} \cos \left(\theta_{T x}\right)+\frac{\left|\vec{v}_{R x}\right|}{c} \cos \left(\theta_{R x}\right)
$$

The Doppler spectrum for this signal is an impulse at $f_{d}$. Assuming the vehicles' positions and velocities are being explicitly modeled, the necessary parameters can be computed geometrically.

\begin{tabular}{ll}
\hline Parameter & Notation \\
\hline Receiver position & $P_{R x}$ \\
Transmitter position & $P_{T x}$ \\
Receiver velocity & $\vec{v}_{R x}$ \\
Transmitter velocity & $\vec{v}_{R x}$ \\
\hline
\end{tabular}

Table 2: Parameters of Line of Sight Doppler Model

\subsubsection{LOS Existence Status}

Whether a LOS path exists, between transmitting and receiving vehicles, determines whether a dominant frequency component (the LOS Doppler Spectrum) will be added in this path or not at run time.

Rather than modeling the exact LOS status which are less predicable, we studied its existence pattern statistically among channel measurement traces [6]. A trace is labeled as LOS-evident if: (a) there exists a frequency component with high power contributing in received signal; or (b) there exists very few other components that are comparable (detected as peaks). The intuition is that, only one LOS path may exist in any situation, and it should manifest as the only one dominating component if it exists. Figure 5 shows the pattern extracted from traces.

The V2V LOS status can then be modeled using a twostate Markov model, with state-1 indicating there exists

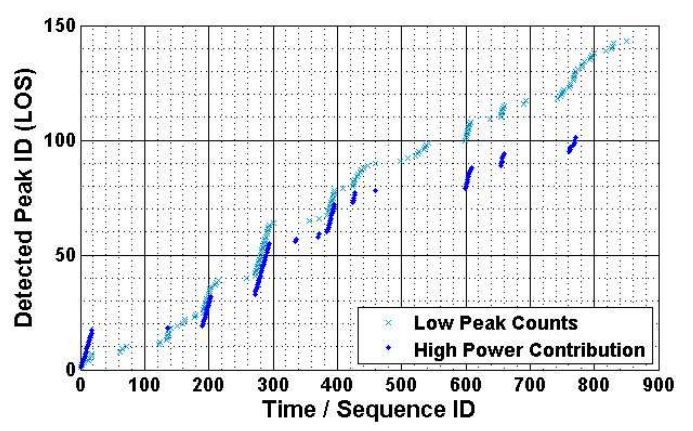

Figure 5: Dominant Components (LOS)

$L O S$, and state-0 if not (a.k.a. non-LOS or NLOS). Switching between states is regulated by parameters in the state transition matrix.

\subsection{Geometry-based Fading Doppler Spectrum}

The geometry-based fading Doppler spectrum describes the aggregate signal received by indirect paths reflected by objects in the environment. Here we consider the model described in [4] as an example. Fading effects from roadside scatterers are captured in this model, for two vehicles driving on a straight street, where scatters are road-side trees and buildings. The model's parameters are given in Table 3. Other sophisticated v2v channel models such as [5] have very similar parameter sets.

\begin{tabular}{ll}
\hline Parameter & Notation \\
\hline Transmitter (Tx) and receiver (Rx) velocity & $\vec{v}_{T x}, \vec{v}_{R x}$ \\
Distance between Tx and Rx & $d_{t r}$ \\
Density of road-side scatterers & $\rho$ \\
(Mean) distance from road side to scatterers & $d_{s 2 e}$ \\
Width of road lanes & $d_{l a n e}$ \\
Number of lanes "above" (left of) Tx and Rx & $N_{a}$ \\
Number of lanes "below" (right of) Tx and Rx & $N_{b}$ \\
\hline
\end{tabular}

Table 3: Parameters of Geometrical V2V Model

An example of estimating vehicular channel parameter is shown in $\S 4.2$. Our goal is to identify practical method of estimating parameters values with high accuracy, based on our classification in Table 3

\subsection{Finer Granularity Fading (Spectrum De- smoothing)}

The geometry-based model describes an average or expected fading Doppler spectrum (envelope), based on the estimated density of scatterers. We refine this by estimating the properties of specific segments of roadway. In all of these cases, the regions over which such estimates are made are necessarily much larger than the size of an individual scattering feature, indicating that the modeled spectrum will be significantly smoothed relative to reality. The actual fading spectrum used in simulation is then filtered with a random variable, as suggested in [8] to approach actual randomness in scatterer density at a finer granularity.

We have developed models for (a) characterizing the nonsmoothness of observed Doppler spectra, and (b) reintroducing realistic variation in modeled scattering Doppler spec- 
tra. First, we analyzed the non-smoothness by detecting peak locations in measured Doppler spectrum. Since each peak represents strong reflections from scatterers, the spacing between peak locations indicates the spatial distribution pattern of scatterers along the road, and may include mobile scatterers as well. When calculating the channel response, a similar peak spacing pattern is used for filtering sequences, which represent the desired non-smoothness in particular environment. The filtering sequences essentially reallocate powers among different frequency shifts, and generate more realistic fading effects in traces. More accurate modeling of mobile scatterers is still a challenging problem yet to be explored.

Figures $8 \mathrm{~b}$ and $8 \mathrm{c}$ show the effects of simple Gaussian noise (b), and a physically-motivated energy coalescing process (c). Relevant parameters are shown in Table 4. Note that these approaches model the observed effect at the signal level; we have not yet developed predictive models for this variation.

\begin{tabular}{lll}
\hline Model & Parameter & Notation \\
\hline Gaussian & Standard error & $\sigma^{2}$ \\
Coalescing & Peak component number & $n_{o}, n_{f}$ \\
Coalescing & Peak component spacing & $\delta f_{\text {peak }}$ \\
Coalescing & Peak energy fraction & $p_{\text {peak }}$ \\
\hline
\end{tabular}

Table 4: Parameters of Scattering Granularity Models

In this paper we do not attempt to explicitly model mobile scatterers, i.e. other vehicles on the road. Modeling of mobile scatterers is difficult due to lack of exact information of their high dynamics over space and time. As we do not have such data available, we settle for implicitly modeling mobile scatterers as part of the random "error" in the scattering Doppler spectrum.

\subsection{Component Contributions}

The Doppler spreading spectra for both the line of sight and scattered signals contribute to the received signal. While total received power is determined by large-scale path loss, the relative weight (power contribution) among each components need to be configured. The channel effects of each component - tap weight contributions in our realization must be scaled to represent the magnitude of the channel gain (loss) for each. The range of relative weight can be obtained from measurements in specific environment, and then be applied to simulation of similar scenarios.

For the LOS component, we model these magnitudes as (1) an absolute path loss magnitude, and (2) a relative lineof-sight magnitude. In this paper, we do not attempt to evaluate path loss models - we are using the same (measured) path loss value with both the modeled and measured fading processes in our evaluation. For fading components, we learned the relative power ratio for specific vehicular environments from trace study in [6], and applied the same value in implementation and evaluation.

\section{MODEL IMPLEMENTATION}

The system described in $\S 2$ is implemented as a new V2V channel model on the Wireless Network Emulator [7]. Two major modules were added, for V2V LOS and fading/scattering.

\subsection{V2V Line of Sight Module}

The LOS status model has two configurable parameters: 1) $t_{N L O S}$ : the average duration of $N L O S$ period; and 2) $p_{\text {block }}$ : the probability of losing LOS. Another variable $t_{L O S}$ records the accumulated duration of LOS period, and determines pblock accordingly: the longer a LOS period is, the higher $p_{\text {block }}$ should be.

For the configurable parameters in this model, we tried to find reasonable typical values representing studied suburban area from traces statistics.

As shown in Figure 5, consecutive samples of LOS component appear as line segments, with gaps indicating NLOS periods. The value and range of $t_{L O S}$ and $t_{N L O S}$ are then determined by the average length of continuous LOS segments, and gaps in between.

\subsection{Geometry-based Fading and Scatterer Es- timation}

As discussed in $\S 3$, accuracy in density estimation is a critical set of parameters in the $\mathrm{V} 2 \mathrm{~V}$ fading model [4]. Instead of using default value suggested in that paper, we added a scatterer estimation module to better model roadside scatterers.

For the purposes of this paper, we consider two types of scatterer: trees and man-made structures. Some propagation studies have attempted to identify and model every specific object in the region of interest, but this is impractical at the scale of a meaningful vehicular network. We are therefore interested in approximations that can be applied to large areas, using existing publicly-available data, in an automated way.

We explored several alternatives, and were able to achieve the best accuracy by extracting relevant features from aerial photographs. While the availability and quality of imagery varies by region, $1 \mathrm{~m} \times 1 \mathrm{~m}$ digital aerial orthophotography is available for most of the United States [9]. Using established spectral signature criteria [10], the type of land cover in each pixel can be estimated. Figure 6 shows an example classification map generated for central Pittsburgh. The location and density of roadside scatters and road (lane) dimensions can be estimated from the land cover classification results, combined with explicit road information from U.S. Census data [11].

The accuracy of mapping is limited by image quality and classification algorithms. However, this general approach proves effective in practice, and accuracy can be improved with better inputs. Although the processing time for a large area requires intensive computations, the whole process is only executed once for static objects.

For the area we are studying, the best aerial maps available are from National Agricultural Imagery Program [9], The most recent photographs in this area are 4-band (RGB plus infrared), with an absolute position accuracy of $\pm 6 \mathrm{~m}$.

For any given road segment, the scatterer distribution model calculates roadside scatterer (building and trees) density. Considering the fact that locations of building and trees are stationary in general, the calculation is performed off-line for any given area of interest. At run time, exact scatterer density along the route (at given locations) can be obtained quickly by a simple table (map) lookup.

Figure 7 shows sample roadside scatterer densities generated with a given route. Using these better-estimated parameters, fading models can generate more realistic location- 


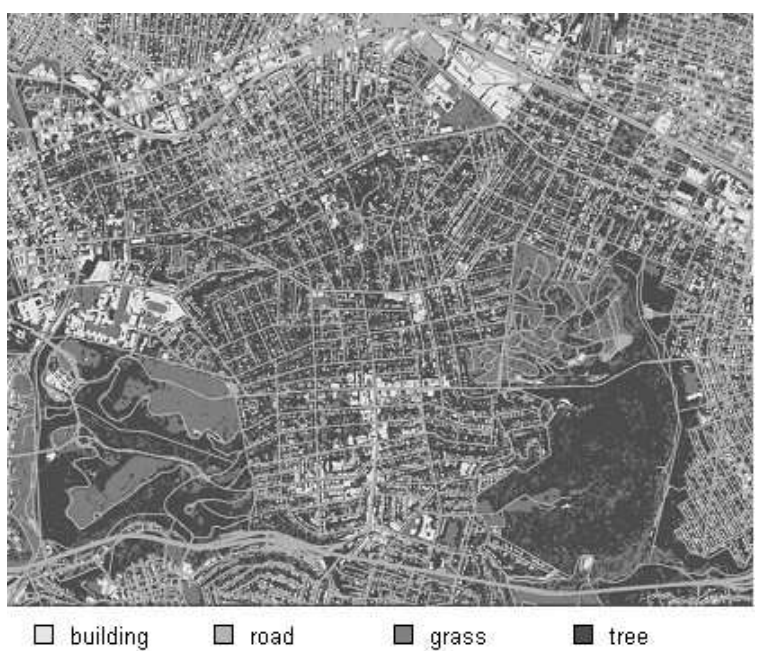

Figure 6: Classified Map

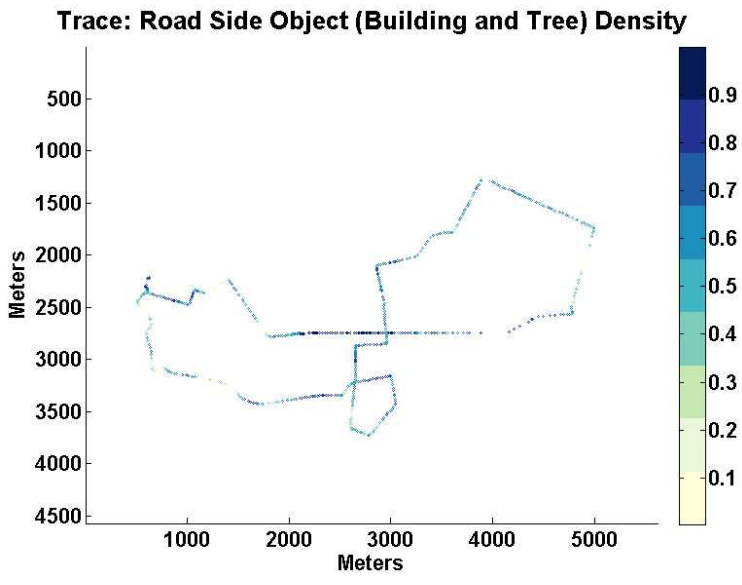

Figure 7: Roadside Scatterer Density

specific Doppler spectrum. The benefits are shown in 5, when compared against default parameter values.

\section{EVALUATION}

In this section, we show how the proposed realistic V2V channel model helps to improve realism in emulation. More specifically, the impact of accurate parameter estimation is examined in both physical layer propagation emulation, and end to end link layer packet delivery performance.

\subsection{Doppler Spectrum Generation}

The implemented channel model is able to construct and emulate V2V channel components including LOS existence, accurate fading effects from static roadside scatterers, in addition to path loss and simple mobile-to-stationary fading models. Figure 8a shows a reconstructed Doppler spectrum that includes the $\mathrm{V} 2 \mathrm{~V}$ fading envelope, as well as a LOS component. Compared with measured Doppler spectrum shown in Figure 8d, the fading envelope represents averaged scattering effects over space. Figure $8 \mathrm{c}$ shows the Doppler spectrum filtered with the realistic energy coalescing process, to represent finer granularity fading, as described in $\S 3.3$.

\subsection{Doppler Spectrum Similarity}

In the first experiment, we compare the difference between simulated fading spectrum and measured spectrum in physical world. We first collect the exact geographic and mobility information in measurement study [6], where 889 Doppler spectra (called $M_{1}-889$ ) are recorded with each representing a $1.5 \mathrm{~s}$ continuous-wave measurement trace. Using the geographic scatterer density estimates, we generated a corresponding simulated Doppler spectrum for each trace $\left(E_{1}-889\right)$ using location-specific parameter estimates as described in $\S 4.2$. For comparison, an additional set of spectra (called $\left.D_{1}-889\right)$ are generated using averaged (estimated) parameter values for the entire region.

Now we compare $D_{1}-889$ and $E_{1}-889$ against $M_{1}-M 889$ 2 to show the closeness between simulated spectrum and measured spectrum.

The spectrum similarity is quantified using the KullbackLeibler divergence [12], by normalizing each spectrum as a probability distribution: $D_{i}, E_{i}$, and $M_{i}$, where $i$ is 1 to 889 . If frequency sample points are denoted as $f_{j}$, the KullbackLeibler divergence of two spectra is then defined as:

$$
d_{K L}\left(E_{i} \| M_{i}\right)=\sum_{j} E_{i}\left(f_{j}\right) \ln \frac{E_{i}\left(f_{j}\right)}{M_{i}\left(f_{j}\right)}
$$

and $d_{K L}\left(D_{i} \| M_{i}\right)$ is defined similarly. The Kullback-Leibler divergence value is lower if two distributions (spectra) are similar.

The spectrum similarities $d_{K L}\left(D_{i} \| M_{i}\right)$ and $d_{K L}\left(E_{i} \| M_{i}\right)$ along a measured route are shown in Figure 9. Figure 10 shows empirical CDF(Cumulative Distribution function) of the spectrum divergence for the two estimation methods.

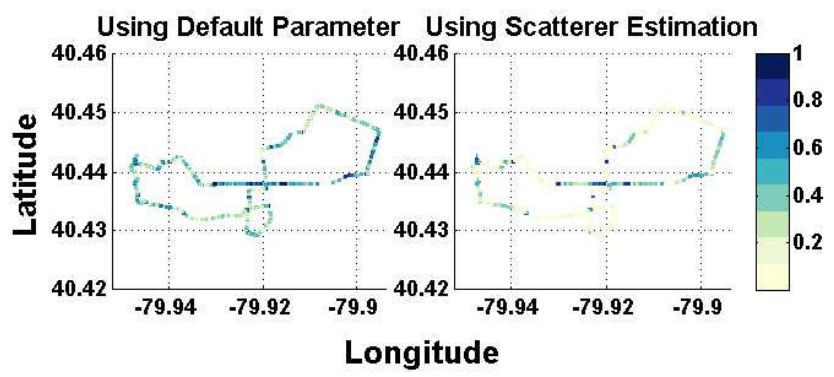

Figure 9: Spectrum Similarity Comparison on Map

The results show that $d_{K L}\left(E_{i} \| M_{i}\right)$ value is significantly lower, which indicates the emulated fading spectrum is more accurate when location-specific parameter estimates are used.

\subsection{Link Layer Comparison}

Given the significant difference in emulated fading spectrum, we are interested in how the link layer performance would differ when the following channel propagation models are applied in emulation: $E_{i}$ and $D_{i}$, as well as $M_{i}$ as benchmarks.

The experiment is performed with two 802.11a nodes in ad-hoc (IBSS) mode, when these two nodes are isolated from

\footnotetext{
${ }^{2}$ Notice that $D_{1}-889$ and $E_{1}-889$ are basic fading envelopes. Therefore, we applied a smoothing window of $100 \mathrm{~Hz}$ on the measured spectrum and use the result as the fading spectrum envelope $M_{1}-889$.
} 


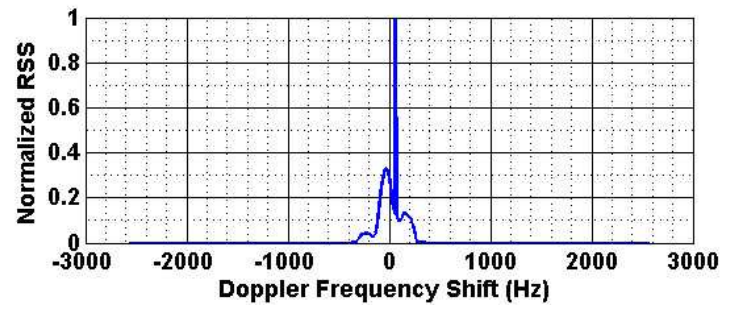

(a) LOS and Fading Envelope

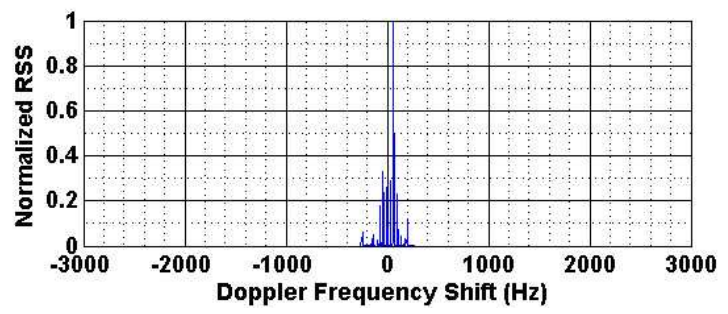

(c) Filtered with Realistic Fading Scatterer

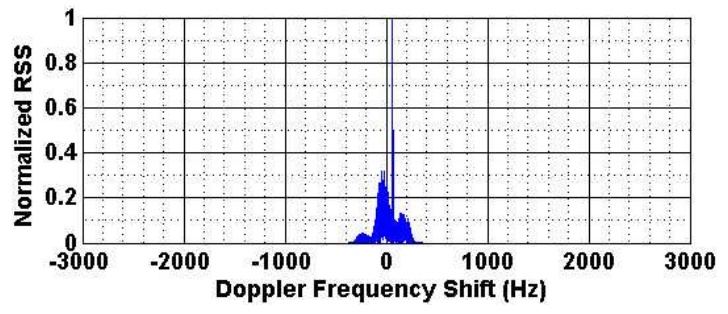

(b) Filtered with Gaussian variates

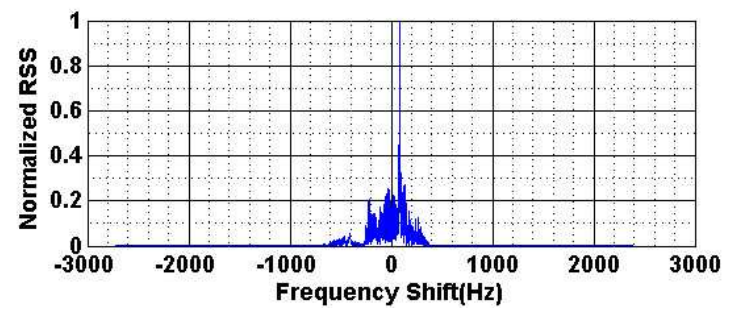

(d) Doppler Spectrum Measurement from Trace \# 100

Figure 8: Generate Channel Doppler Spectrum

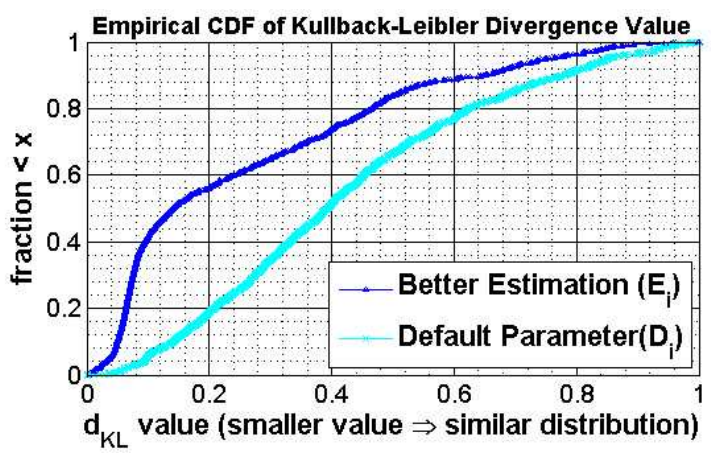

Figure 10: Spectrum Similarity Comparison (CDF)

the environment in emulation (both using $5.2 \mathrm{GHz}$ channel). A simple broadcast ping flood from the source node is used as one-way test traffic, and received packets are captured with tcpdump on the target node. The measurement is repeated using all $E_{i}, D_{i}$, and $M_{i}$ as fading spectra.

Figure 11 shows the packet-delivery-ratio (pdr) measurement for an experiment lasting for 100 seconds. The distance between two mobiles ( $T x$ and $R x$ ) varies as they travel along a predefined route, with different speeds and start times. The measured PDR varies significantly over time as the path loss and LOS change, but the results for the different spectra in any given time window are fairly close. We believe this is because that the large-scale path loss dominates the received signal strength, which in turn dominates the PDR results.

Further examination is needed to understand how different small-scale fading spectrum would impact packet reception rates, both on average and at smaller time scales, when the impact of large-scale path loss is similar. It is also anticipated that the path loss and LOS models can be refined using location-specific information, much as we have done with the small scale fading model.

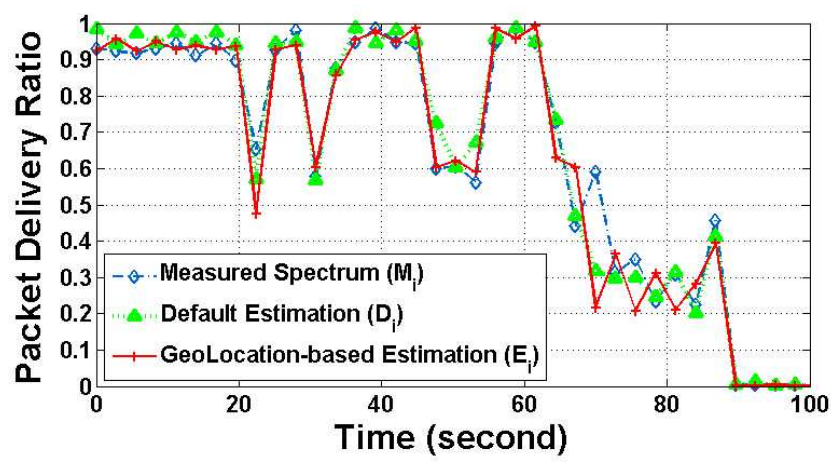

Figure 11: PDR Comparison

\section{RELATED WORK}

Research related to simulation of vehicle-to-vehicle channels includes channel characterization, channel modeling, and experimental platforms for v2v communication. Here is a brief overview of most relevant works.

Channel Characterization of $\mathrm{V} 2 \mathrm{~V}$ propagation has been performed in various environments [13]. Different aspects of channels properties have been investigated in measurementbased studies, e.g. narrowband Doppler shift [6], wideband impulse response [14] and MIMO channels [15]. Other experiments explore LOS(and NLOS) effects in propagation channels [16] and shows significant impact on packet reception [17] in vehicular channels.

Channel Models have been proposed to represent corresponding propagation features, including multi-path(multitap) with path delay [15], and fading models. Geometrybased fading models make assumptions of scatterer distribution pattern, such as a single ring [18] and a double ring [8]. Measurements $[6,14]$ show that fading effects in V2V channel have distinct features beyond what generalized mobileto-mobile fading models usually capture. Models with better assumptions on scatterer patterns have been developed, for 
both LOS [19] and fading effects [4,5]. Finding accurate values for model parameters for any specific environment is a significant challenge with any of these models.

V2V Experimental Platforms: General wireless network simulators $[1,2]$ target network-scale experiments, and provide limited support for v2v channel properties. Similarly, vehicular network simulators $[20,21]$ focus on modeling of (vehicular) traffic generation and mobility to improve realism, but do not significantly consider the radio channel implications. [22] shows that the realism in channel modeling plays a critical rule in achieving high approximation in simulation. Real-time emulation platforms [3] are also available for general wireless experiments, but were not designed specifically for $\mathrm{v} 2 \mathrm{v}$ communications. Vehicular testbeds, such as The Connected Vehicle Test Bed [23] and [24] provide the most real environment for experiments, but less control and almost no repeatability are available.

\section{CONCLUSION}

In this paper, we focus on improving realism in V2V channel emulation and parameter accuracy. Two new emulation models are designed and implemented to represent realistic V2V LOS and roadside scattering features. High accuracy in model parameter estimation is achieved by utilizing aerial photos as well as trace-based study.

\section{ACKNOWLEDGMENTS}

This research was funded in part by NSF under award number CNS-0434824, and in part by the Air Force Research Laboratory under award number FA89750.10.1.0232. We would like to thank Prof. Lin Cheng and Prof. Daniel Stancil for discussion on channel models, and providing empirical V2V traces collected via their project. Also, a special thanks to Dr. Xiaoxiao Li, for her valuable input on utilizing aerial photographs.

\section{REFERENCES}

[1] "Network Simulator: ns-3," http://www.nsnam.org/.

[2] "QualNet," http://www.scalable-networks.com.

[3] X. Wang, K. Borries, E. Anderson, and P. Steenkiste, "Network-scale emulation of general wireless channels," in Vehicular Technology Conference (VTC Fall), 2011 IEEE, September 2011, pp. $1-5$.

[4] L. Cheng, F. Bai, and D. D. Stancil, "A new geometrical channel model for vehicle-to-vehicle communications," in Proc. IEEE Antennas and Propagation Society Int. Symp. (APS/URSI), 2009.

[5] J. Karedal, F. Tufvesson, N. Czink, A. Paier, C. Dumard, T. Zemen, C. Mecklenbrauker, and A. Molisch, "A geometry-based stochastic mimo model for vehicle-to-vehicle communications," IEEE Transactions on Wireless Communications, vol. 8, no. 7, pp. $3646-3657$, July 2009.

[6] L. Cheng, B. Henty, D. Stancil, F. Bai, and P. Mudalige, "Mobile vehicle-to-vehicle narrow-band channel measurement and characterization of the 5.9 ghz dedicated short range communication (dsrc) frequency band," IEEE Journal on Selected Areas in Communications, vol. 25, no. 8, Oct. 2007.

[7] G. Judd and P. Steenkiste, "A software architecture for physical layer wireless network emulation," in WiNTECH, Los Angeles, 2006.
[8] C. Patel, G. Stuber, and T. Pratt, "Simulation of Rayleigh-faded mobile-to-mobile communication channels," IEEE Transactions on Communications, vol. 53, no. 11, pp. 1876 - 1884, Nov. 2005.

[9] Aerial Photography Field Office, "National agricultural imagery program," http://www.fsa.usda.gov/FSA/ apfoapp?area $=$ home\&subject $=$ prog\&topic $=$ nai, 2010.

[10] T. Blaschke, "Object based image analysis for remote sensing," ISPRS Journal of Photogrammetry and Remote Sensing, vol. 65, no. 1, pp. 2-16, 2010.

[11] U.S. Census Bureau, "TIGER/Line Shapefiles," 2011.

[12] S. Kullback, "Information theory and statistics," John Wiley and Sons, NY, 1959.

[13] A. Molisch, F. Tufvesson, J. Karedal, and C. Mecklenbrauker, "Propagation aspects of vehicle-to-vehicle communications - an overview," in IEEE Radio and Wireless Symposium, Jan. 2009.

[14] A. Paier, J. Karedal, N. Czink, H. Hofstetter, C. Dumard, T. Zemen, F. Tufvesson, A. F. Molisch, and C. F. Mecklenbräuker, "Car-to-car radio channel measurements at $5 \mathrm{GHz}$ : Pathloss, power-delay profile, and delay-doppler spectrum," in Proc. 4th Int. Symp. Wireless Communication Systems, 2007.

[15] G. Acosta-Marum and M. Ingram, "Doubly selective vehicle-to-vehicle channel measurements and modeling at 5.9 ghz," in Proc. Int. Symp. Wireless Personal Multimedia Commun, 2006.

[16] R. Meireles, M. Boban, P. Steenkiste, O. Tonguz, and J. ao Barros, "Experimental study on the impact of obstructions in vehicular ad hoc networks," in The 2010 IEEE Vehicular Networking Conference, 2010.

[17] F. Martelli, M. Renda, G. Resta, and P. Santi, "A measurement-based study of beaconing performance in ieee 802.11 p vehicular networks," in INFOCOM, 2012.

[18] A. S. Akki and F. Haber, "A statistical model of mobile-to-mobile land communication channel," IEEE Transactions on Vehicular Technology, vol. 35, no. 1, pp. 2-7, 1986.

[19] E. Giordano, R. Frank, G. Pau, and M. Gerla, "Corner: a realistic urban propagation model for vanet," in Wireless On-demand Network Systems and Services (WONS). IEEE, 2010, pp. 57-60.

[20] R. Mangharam, D. Weller, R. Rajkumar, P. Mudalige, and F. Bai, "Groovenet: A hybrid simulator for vehicle-to-vehicle networks," in Third Annual International Conference on Mobile and Ubiquitous Systems, 2006.

[21] M. Behrisch, L. Bieker, J. Erdmann, and D. Krajzewicz, "Sumo - simulation of urban mobility: An overview," in The Third International Conference on Advances in System Simulation, Barcelona, Spain, October 2011, pp. 63-68.

[22] E. M. Belding-Royer, "Wireless networking outside of the simulator," in Proc. 29th Annual IEEE Int Local Computer Networks Conf., 2004.

[23] "The connected vehicle test bed," http://www.its.dot. gov/connected_vehicle/technology_testbed2.htm.

[24] M. Cesana, L. Fratta, M. Gerla, E. Giordano, and G. Pau, "C-vet the ucla campus vehicular testbed: Integration of vanet and mesh networks," in European Wireless Conference (EW). IEEE, 2010, pp. 689-695. 